\title{
Experimental and Numerical Investigation on the Transport Characteristics of Particle-Fluid Mixture in Y-Shaped Elbow
}

\author{
Qiong Hu ${ }^{1,2,3}$, Li Zou ${ }^{2,4, *}$, Tong Lv ${ }^{1,2}$, Yingjie Guan ${ }^{2}$ and Tiezhi Sun ${ }^{4}$ \\ 1 School of Mechanical and Electrical Engineering, Central South University, Changsha 410083, China; \\ huqiong@csu.edu.cn (Q.H.); lvt_csu@163.com (T.L.) \\ 2 State Key Laboratory of Deep Sea Mineral Resources Development and Utilization Technology, \\ Changsha 410012, China; guanyj93@163.com \\ 3 Changsha Research Institute of Mining and Metallurgy, Changsha 410012, China \\ 4 School of Naval Architecture, Dalian University of Technology, Dalian 116024, China; suntiezhi@dlut.edu.cn \\ * Correspondence: lizou@dlut.edu.cn; Tel.: +86-0411-8470-6373
}

Received: 13 July 2020; Accepted: 25 August 2020; Published: 2 September 2020

\begin{abstract}
The Y-shaped elbow is used as a connecting pipe between the buffer and the lift pipe in the deep-sea mining system. After being mixed with seawater in the Y-shaped elbow, nodule particles are lifted to the sea surface mining ship via the lift pump. In this paper, we employ a computational fluid dynamics and discrete element coupled method (CFD-DEM) to study the characteristics of particle transport in the Y-shaped elbow. Considering a large diameter of the particles, we discuss the behavior of particles and fluid under different conveying velocities. In addition, the simulation was verified based on the experiment. The results show that the simulation agrees well with the experiment. On this basis, the distribution and motion characteristics of the particles in the Y-shaped elbow were obtained. The interaction between fluid and particles is also discussed. These findings suggest that the particles can be successfully transported when the pump runs at medium to high frequencies. The particles are basically moving along the pipe wall and slower than the fluid flow. Moreover, it was found that the particle motions are more complex with the increasing of conveying velocities, and it is closely related to the secondary flow of fluid. Some suggestions on the actual particle transportation can be put forward based on the research in this paper.
\end{abstract}

Keywords: Y-shaped elbow; deep-sea mining; particle-liquid flow; CFD-DEM coupled calculation

\section{Introduction}

With the development of deep-sea mining, the conveying system is becoming increasingly important. As shown in Figure 1, the currently used deep-sea mining model mainly includes: tracked mining vehicle, flexible hose, buffer, rigid lift pipe, lift pump, and sea surface mining vessel. During the process, the nodules collected by the vehicle are transported by seawater through the hose into the buffer, which is a frame structure constructed of angle iron. As can be seen from inside the buffer, the collected mixture of nodules and seawater is put into the Y-shaped elbow through the feeding machine under the silo, seawater is pumped into the pipe through the other inlet of the Y-shaped elbow by the lift pump. The mixture of nodule particles and seawater is conveyed to a surface mining vessel via the lift pipe. Different motion states of nodule particles can be caused due to different conveying velocities. If particle leakage or even accumulation or blockage occurs, not only the efficiency of nodule transport is reduced, but also the safety of the entire pipeline system is threatened. Therefore, it is important to study the transport characteristics of large particles in the Y-shaped elbows under different working conditions. 


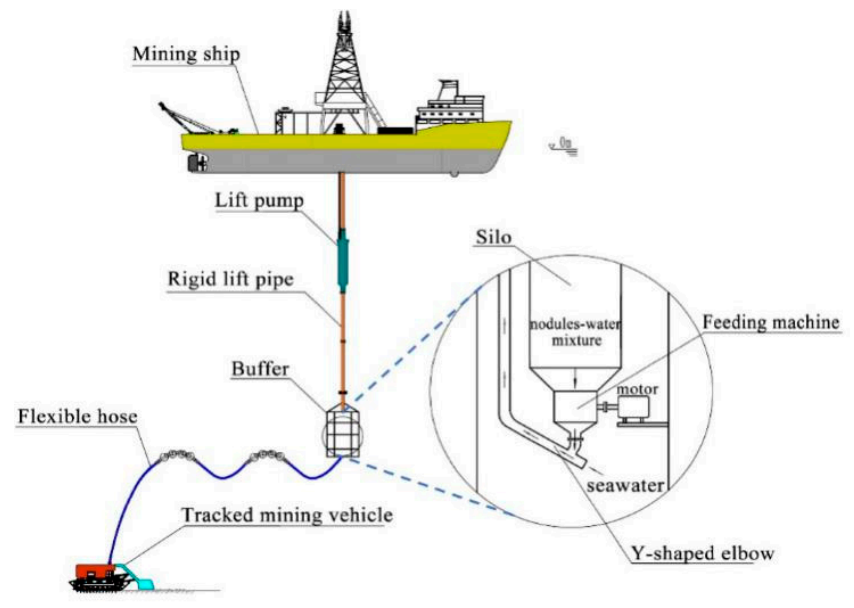

Figure 1. Schematic diagram of deep-sea mining system and Y-shaped elbow.

In recent years, the Euler-Euler and Euler-Lagrange methods have been used as important numerical simulation methods to investigate the particle-liquid flow in pipelines. The main idea of the Euler-Euler two-fluid model is to treat the particles in the mixed fluid as a continuous medium, so that both the particles and fluid maintain interpenetrating continua. Compared with the Euler-Lagrange method, the Euler-Euler method requires much less computational resources and some characteristics of discrete particle phase are omitted. This model is mainly used in applications where the concentration of discrete phases is relatively high. Therefore, it can be used for the study of small particles (below $1 \mathrm{~mm}$ ) or slurry transport [1-5]. However, when the particle concentration is low so that the interactions between particles and particles, particles and fluid cannot be ignored, the Euler-Lagrange method is more in line with the computational requirements, in which particles are solved by Newton's law of motion in the Lagrange coordinate system as the discrete phase while fluid is solved as the continuous phase in the Euler coordinates. The method takes the characteristics of the discrete particle phase into account. Therefore, the results agree better with the real state of the particle motion in the fluid. The Euler-Lagrange method is gradually applied to the analysis of particle motion behaviors, particle transportation, pipe wear, etc. [6-9].

With the increasing requirement of calculation, in order to combine with the advantages of the Euler-Lagrange method and fully consider the movement behavior of particles in the process of solid-liquid two-phase flow transportation, more and more researchers choose the coupling simulation method of discrete element method (DEM) and computational fluid dynamics (CFD). In 1979, the discrete element method (DEM) was proposed by American scholars Cundall and Strack [10], and gradually applied to study slurries transportation, mixture flow in dam break, particle behavior in medical research and so on [11-13].

The particle solid-liquid two-phase flow in the sludge discharge pipe was simulated by the discrete element software EDEM [14]. The distribution rule of particle groups at the connection of the pipe and the flange was studied. The CFD-DEM coupling method based on the open source software package OpenFOAM and LIGGGHTS was analyzed and described. The simulation of solid-liquid two-phase flow of fluidized bed, hydrocyclone and other test cases was carried out to verify the applicability of the method, which provided a new method for the future research direction [15]. The solid-liquid mixing process under the action of turbine based on CFD-EDM was simulated. The concentration distribution of suspended particles of the experiments proved the accuracy of the method [16]. The distribution and movement state change of coarse particles in the horizontal pipeline under different transportation conditions was analyzed by the CFD-DEM coupling method. The influence mechanism of transportation conditions on the solid-liquid flow of coarse particles was found. At the same time, the interaction between particles had the most important impacts on the suspension of 
particles. It is obvious that CFD-DEM coupling calculation could get more comprehensive flow field information and particle motion law, which has great application prospects [17].

In addition to the simulation methods, many achievements about characteristics of particle transportation in kinds of pipe forms have been made. To reveal the regularity of movement of nodule particles in vertical lifting pipelines, the trajectory, lifting and settlement characteristics of the particles were studied experimentally, and the effect of vertical swinging on the particle transport in the pipeline was also investigated [18-22]. In the horizontal pipelines, the researchers have undertaken significant investigations on slurry transport [17,23-27]. As for elbows, inclined and tee pipes, the researchers have undertaken significant investigations on slurry transport [28,29] and the wear of the key parts of the pipeline caused by microsized particles and solid-liquid movement analysis [30-34]. However, the transport characteristics of large particles in the Y-shaped elbow of the deep-sea mining system remain unclear. To the author's knowledge, little research has been devoted to the Y-shaped elbow with two inlets and in which the particles are mixed with the fluid and transported.

Based on the research trends and the improvement of current deep-sea mining system, we implemented a coupled CFD-DEM simulation of particle-fluid mixture flow in Y-shaped elbow using the commercial software Ansys Fluent and EDEM in this paper. The experiment of particle transportation was also carried out to verify this method. The purpose of this study was to investigate the motion characteristics of the fluid and particles in the Y-shaped elbows under different working conditions. Furthermore, the conditions for the safe transportation of the particles will be put forward. The CFD-DEM simulation verified in this paper will be applied to the study of various pipe forms of the subsequent conveying system.

\section{Experimental Platform}

To verify the simulation model, an experimental platform was built as shown in Figure 2. The experiment system aims to form a particle-transporting loop. At the beginning, nodule particles are put into the feeding pipe through the hopper and mixed with the water from the regulated tank. Then the mixture flows into the Y-shaped elbow in the pressure-bearing tank. After that, the mixture flow is lifted through the lifting pipe by the centrifugal pump and finally returns to the hopper inlet. The purpose of the regulated water tank is to regulate the pressure of the pipeline and the pressure-bearing tank. After the system pressure remains stable, the water-supply valve will be closed. During the experiment, the transporting characteristics of particles and water in the Y-shaped elbow can be recorded through the observation window. Some experimental parameters will provide reference for the simulation.

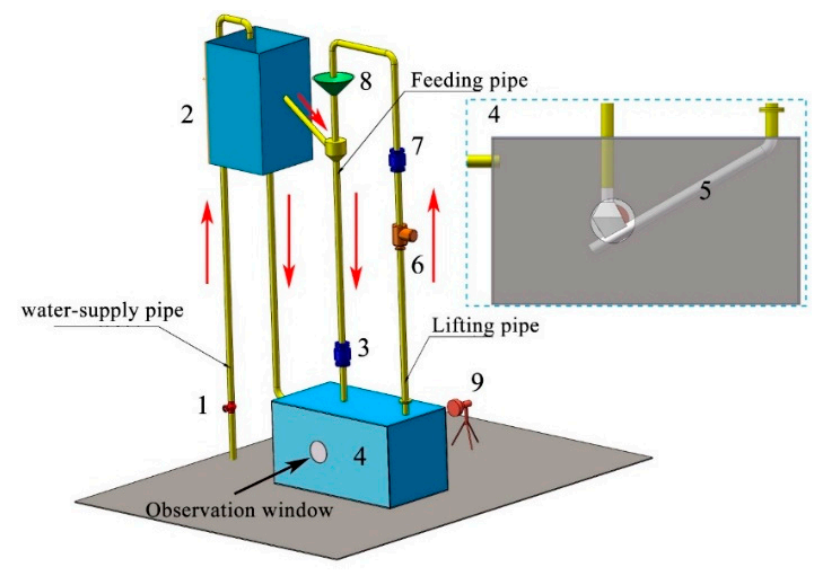

Figure 2. Schematic diagram of the experimental platform for particle transport in Y-shaped elbow (1. Water-supply valve, 2. Regulated water tank, 3 (7). Flowmeter, 4. Pressure-bearing tank, 5. Y-shaped elbow, 6. Centrifugal pump, 8. Feeding hopper and 9. Light bulb). 
The measurement and control subsystem mainly includes two parts: flow measurement and pump speed regulation. Due to the difference of the flow rate between the feeding pipe and the lifting pipe, it is necessary to install the electromagnetic flowmeter in the feeding pipe and the lifting pipe. The frequency converter can control the speed of the centrifugal pump motor to realize different conveying velocity in the pipeline. During the test, the particle running state in the pipeline was captured by a high-speed camera. The detailed information of the main instruments and equipment used in the test is shown in Table 1.

Table 1. Detailed information of the main instruments and equipment.

\begin{tabular}{|c|c|c|}
\hline Name & Model & Parameters \\
\hline Centrifugal pump & ISG 50-160 (I) A & $\begin{array}{l}\text { The rated power is } 3 \mathrm{~kW} \text {, the rated flow is } 29 \mathrm{~m}^{3} / \mathrm{h} \text {, the lift is } \\
16 \mathrm{~m} \text {, the rotational speed is } 2900 \mathrm{r} / \mathrm{min} \text { and the } \\
\text { corrosion-resistant mechanical seal is adopted. }\end{array}$ \\
\hline $\begin{array}{l}\text { Electromagnetic } \\
\text { flowmeter }\end{array}$ & ZJLDG-50 & $\begin{array}{l}\text { The nominal diameter is } 50 \mathrm{~mm} \text {, the working range is } \\
0.7-106 \mathrm{~m}^{3} / \mathrm{h} \text {, the working pressure is } 1.6 \mathrm{MPa} \text {, the working } \\
\text { temperature is under } 80^{\circ} \mathrm{C} \text {, the electrode is made of } 316 \mathrm{~L} \\
\text { stainless steel and the lining is polyurethane. }\end{array}$ \\
\hline $\begin{array}{l}\text { Frequency } \\
\text { converter }\end{array}$ & $\begin{array}{l}\text { Emerson-Enydrive } \\
\text { TD3000 }\end{array}$ & $\begin{array}{l}\text { The suitable motor power is below } 7.5 \mathrm{~kW} \text {, the rated voltage is } \\
380 \mathrm{~V} \text {, the output frequency is } 0-400 \mathrm{~Hz} \text {, four digit digital } \\
\text { display, with Chinese and English } \mathrm{LCD} \text { display, the } \\
\text { installation mode is wall mounted. }\end{array}$ \\
\hline $\begin{array}{l}\text { High speed } \\
\text { camera }\end{array}$ & Phantom VEO $410 \mathrm{~L}$ & $\begin{array}{l}\text { The full frame resolution is } 1280 \times 800 \text {. The full frame } \\
\text { shooting rate is } 5200 \text { frames/S. In this state, } 9.6 \mathrm{~s} \text { can be } \\
\text { recorded. The minimum exposure time is } 1 \mu \mathrm{s} \text {, the maximum } \\
\text { shooting speed is } 600,000 \text { frames/s, the number of pixels is } \\
1,024,000 \text {, and the pixel size is } 20 \mu \mathrm{m} \text {. }\end{array}$ \\
\hline
\end{tabular}

\section{Numerical Morphology}

\subsection{Fluid and Particle Equations}

The Reynolds-averaged Navier-Stokes equation is applied to the fluid solved in Fluent, which consists of two parts:

(1) Continuity equation

$$
\frac{\partial\left(\boldsymbol{u}_{i}\right)}{\partial x_{i}}=0
$$

(2) Momentum equation

$$
\frac{\partial \rho \boldsymbol{u}_{i}}{\partial t}+\frac{\partial\left(\rho \boldsymbol{u}_{i} \boldsymbol{u}_{j}\right)}{\partial x_{j}}=-\frac{\partial P}{\partial x_{i}}+\frac{\partial\left(\boldsymbol{\tau}_{i j}\right)}{\partial x_{j}}+\rho \boldsymbol{g}+F_{p f}
$$

where $\rho$ is the fluid density, $\boldsymbol{u}_{i}$ and $\boldsymbol{u}_{j}$ are the velocity components in Cartesian coordinates $i, j$, respectively, $P$ is the static pressure of fluid, $\tau_{i j}$ is the fluid viscous tensor component, $g$ is the acceleration of gravity and $\boldsymbol{F}_{p f}$ is the interaction forces between continuous and discrete phases.

$$
\boldsymbol{F}_{p f}=\frac{\sum_{i=1}^{k_{c}} f_{f p, i}}{\Delta V_{c}}
$$

where $f_{f p, i}$ stands for the interaction force between the particle $i$ and fluid and $k_{c}$ is the number of particles in a CFD cell of volume $\Delta V_{c}$. 
There are two movements of particles in the fluid: sliding and rolling. Momentum exchange occurs due to the interactions with surrounding particles, pipe wall and fluid. When the particle is calculated in the Lagrange coordinate system, the particle motion obeys Newton's second law. The governing equations for particle $i$ are:

$$
\begin{gathered}
m_{i} \frac{\mathrm{d} \mathbf{v}_{i}}{d t}=\sum_{j=1}^{k_{i}}\left(f_{n, i j}+f_{t, i j}\right)+f_{f p, i}+m_{i} \boldsymbol{g} \\
I_{i} \frac{\mathrm{d} \boldsymbol{\omega}_{i}}{\mathrm{~d} t}=\sum_{j=1}^{k_{i}}\left(\boldsymbol{M}_{\boldsymbol{t}, i j}+\boldsymbol{M}_{r, i j}\right)
\end{gathered}
$$

Here, $m_{i}$ is the particle mass. $v_{i}$ and $\omega_{i}$ are the particle translational and rotational velocities. $k_{i}$ is the number of particles interacting with particle $i . f_{n, i j}$ is the normal contact force between particles $i$ and $j, f_{t, i j}$ is the tangential contact force, $f_{f p, i}$ is the interaction force between the particle $i$ and fluid, which includes pressure gradient force $f_{p g, i}$ and fluid drag force $f_{f d, i} . m_{i} g$ is the gravitational force. $\boldsymbol{I}_{i}$ is the moment of inertia, $\boldsymbol{M}_{t, i j}$ and $\boldsymbol{M}_{r, i j}$ are the tangential and rolling frictional torques acting on the particle $i$ and $j$, respectively. Figure 3 shows the forces and torques of particles during the collision. The Hertz-Mindlin (no slip) model was adopted in this research. In the particle discrete element method, the Hertz contact theory is generally used to calculate the normal force generated by particle contact, while Mindlin-Deresiewicz contact theory was used to calculate the tangential force. The formula about the forces corresponding to the model are listed in Table $2[16,35,36]$.

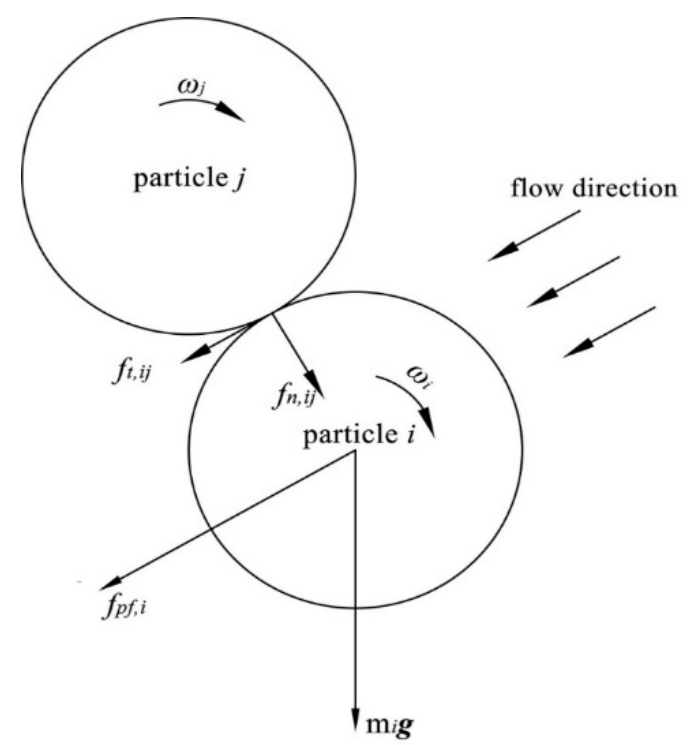

Figure 3. Schematic of the forces acting on particle $i$ from contacting particle $j$.

Table 2. Components of forces and torques acting on particle $i$.

\begin{tabular}{ccc}
\hline Forces and Torques & Type & Formula \\
\hline \multirow{2}{*}{ Normal forces } & Contact & $f_{c n, i j}=\frac{4}{3} E^{*}\left(R^{*}\right)^{\frac{1}{2}} \alpha^{\frac{3}{2}}$ \\
\cline { 2 - 3 } & Damping & $f_{d n, i j}=-2 \sqrt{\frac{5}{3}} \frac{\ln e}{\sqrt{\ln ^{2} e+\pi^{2}}} \sqrt{E^{*} m^{*}\left(R^{*} \alpha\right)^{\frac{1}{2}}} v_{n}{ }^{r e l}$ \\
\hline \multirow{2}{*}{ Tangential forces } & Contact & $f_{c t, i j}=-8 G^{*} \delta \sqrt{R^{*} \alpha}$ \\
\cline { 2 - 3 } & Damping & $f_{d t, i j}=-2 \sqrt{\frac{5}{6}} \frac{\ln e}{\sqrt{\ln ^{2} e+\pi^{2}}} \sqrt{G^{*} m^{*}\left(R^{*} \alpha\right)^{\frac{1}{2}}} v_{t}^{r e l}$ \\
\hline
\end{tabular}


Table 2. Cont.

\begin{tabular}{|c|c|}
\hline \multirow{2}{*}{ Particle-fluid forces } & Pressure gradient force \\
\hline & $f_{f d, i}=\frac{1}{8} \pi C_{D} \rho_{f} d_{i}^{2}\left|\boldsymbol{u}_{f}-\mathbf{v}_{p}\right|\left(\boldsymbol{u}_{f}-\mathbf{v}_{p}\right)$ \\
\hline \multirow{2}{*}{ Torques } & $\boldsymbol{M}_{t, i j}=\boldsymbol{R}_{i} \times\left(f_{c t, i j}+f_{d t, i j}\right)$ \\
\hline & $M_{r, i j}=-\mu_{r} f_{c n, i j} R_{i} \omega_{i}$ \\
\hline \multicolumn{2}{|c|}{ Where: $\frac{1}{E^{*}}=\frac{1-v_{i}^{2}}{E_{i}}+\frac{1-v_{j}^{2}}{E_{j}}, R^{*}=\frac{R_{i}+R_{j}}{R_{i} R_{j}}, G^{*}=\frac{2-v_{i}^{2}}{G_{i}}+\frac{2-v_{j}^{2}}{G_{j}}, m^{*}=\frac{m_{i} m_{j}}{m_{i}+m_{j}}, \mathbf{v}_{n}^{r e l}=\left(\mathbf{v}_{i}-\mathbf{v}_{j}\right) \cdot \frac{\mathbf{r}_{i}-\mathbf{r}_{j}}{\left|\mathbf{r}_{i}-\mathbf{r}_{j}\right|}$} \\
\hline $\mathbf{v}_{t}^{r e l}=\left(\mathbf{v}_{i}-\mathbf{v}_{j}\right) \times \frac{\mathbf{r}_{i}-\mathbf{r}_{j}}{\left|\mathbf{r}_{i}-\mathbf{r}_{j}\right|}, C_{D}=\{$ & $\frac{\mathbf{r}_{i}-\mathbf{r}_{j}}{\mathbf{r}_{i}-\mathbf{r}_{j} \mid}, C_{D}=\left\{\begin{array}{ll}\frac{24}{\operatorname{Re}_{p}}\left(1+0.15 \operatorname{Re}_{p}^{0.687}\right) & , \operatorname{Re}_{p} \leq 10^{3} \\
0.44 & , \operatorname{Re}_{p}>10^{3}\end{array}, \operatorname{Re}_{p}=\frac{\rho\left|\mathbf{u}_{i}-\mathbf{v}_{i}\right| d_{i}}{\mu_{f}}\right.$. \\
\hline
\end{tabular}

\subsection{CFD-DEM Coupling Method}

As mentioned in Section 1, the Euler-Lagrange method is applied to CFD-DEM coupled calculations. The essence is that the particles are calculated and tracked in the interacting flow field of the continuous phase and the discrete phase. A schematic diagram of coupling between Fluent and EDEM is shown in Figure 4. During the calculation, Fluent calculates the fluid firstly, after solving the Reynolds-averaged Navier-Stokes equations, it converges the flow field information to the EDEM. The DEM module in the EDEM calculates the particle forces under the flow field and obtains the particle trajectory. The results are then fed back to Fluent for further iterations until a convergent solution is obtained. In the Eulerian coupling method, considering the volume effect, particles and fluid do not overlap, and each occupies a unique physical space.

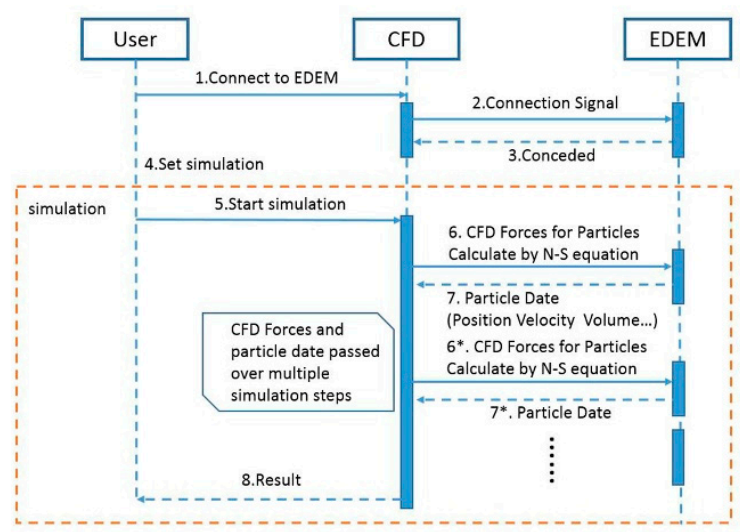

Figure 4. The algorithm of computational fluid dynamics (CFD)-discrete element method (DEM).

\subsection{Y-Shaped Elbow Model and Mesh Generation}

The Y-shaped elbow with $120^{\circ}$ was designed in combination with the design requirements of deep-sea mining pipelines. The specific parameters are shown in Figure 5a. The fluid domain of the pipe was divided into a block-structured grid for making the calculation more accurate and easier to converge. The same trial simulations using grids with different qualities were calculated to select the optimum grid distribution, as shown in Table 3. Comparing the fluid velocity at the outlet, we found that the differences among the results were less than $0.02 \mathrm{~m} / \mathrm{s}$. The results tended to be a fixed value with the grid quantity increasing. Therefore, when the grid quantity reached 130,000 , a satisfactory solution can be guaranteed. One of the meshes is shown in Figure 5. The non-dimensional distance between the first layer of grid nodes and the wall $y+$ considered in this work was about 70 , which was consistent with the k-epsilon turbulence model. 


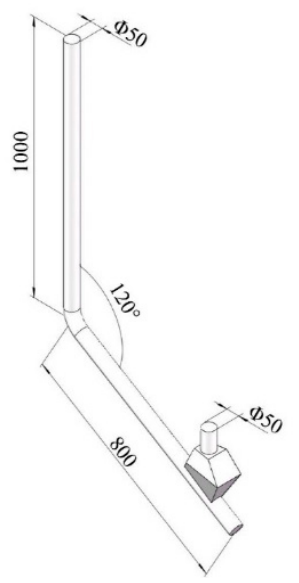

(a)

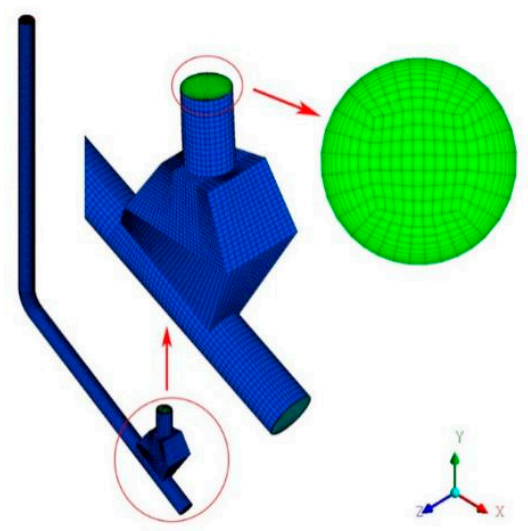

(b)

Figure 5. (a) The schematic of Y-shaped elbow and (b) block-structured grid of the elbow.

Table 3. Grid-independence simulation test.

\begin{tabular}{cccc}
\hline Trial Simulation Conditions & Mesh Quality & Grid Quantity & Average Fluid Velocity at Outlet $(\mathrm{m} / \mathbf{s})$ \\
\hline Particle-inlet $0.5 \mathrm{~m} / \mathrm{s}$, Particle & Coarse & 50,778 & 2.4826 \\
diameter $5 \mathrm{~mm}$ & Medium & 83,488 & 2.4953 \\
Generation rate $0.5 \mathrm{~kg} / \mathrm{s}$ & Fine & 133,952 & 2.4999 \\
Water-inlet $2.0 \mathrm{~m} / \mathrm{s}$, Pressure outlet & Finest & 277,966 & 2.5001 \\
\hline
\end{tabular}

\subsection{Simulation Parameters Setup}

\subsubsection{CFD Settings}

Transient analysis was used for the coupling process. The realizable k-epsilon turbulence model and the standard wall functions were selected for its wide application in industrial flows. The gravitational acceleration was $9.81 \mathrm{~m} / \mathrm{s}^{2}$. The fluid was incompressible in the Y-shaped elbow and water in the Fluent material library was used. The particle inlet and the water inlet were set as the velocity ones and the outlet was set as the pressure outlet. All the inlets and outlet were set constant and mean values to simplify the simulation. The default operating pressure was 1 atmosphere.

As shown in Figure 6, the relationship about the flow rate between particle inlet and outlet was carried out under different pump-running frequencies. The following parameters were selected to simulate the particle transport status of the Y-shaped elbow at low, medium and high velocities, shown in Table 4. $v_{p}$ and $v_{f}$ represent the velocities of particle inlet and water inlet converted from the flowrate under different pump running frequencies.

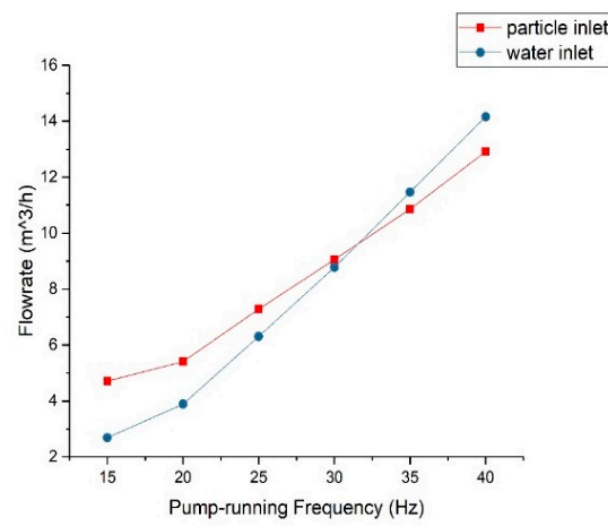

Figure 6. Relationship between flow rates of inlets and pump-running frequencies. 
Table 4. Input parameters in Fluent at different cases.

\begin{tabular}{cccc}
\hline Case & Working Conditions & $v_{p}(\mathrm{~m} / \mathbf{s})$ & $v_{f}(\mathrm{~m} / \mathbf{s})$ \\
\hline 1 & Low $(20 \mathrm{~Hz})$ & 0.76 & 0.55 \\
2 & Medium $(30 \mathrm{~Hz})$ & 1.28 & 1.27 \\
3 & High $(40 \mathrm{~Hz})$ & 1.83 & 2.00 \\
\hline
\end{tabular}

\subsubsection{EDEM Settings}

The particle shape was chosen to be spherical. The diameter of the particle was $5 \mathrm{~mm}$ and particle generation rate was set at $0.4 \mathrm{~kg} / \mathrm{s}$ based on the experiment. Particle velocities kept consistent with the boundary conditions of the particle inlet in Fluent to simplify the simulation. Material properties and related interaction coefficients are shown in Table 5.

Table 5. Material properties of elbow and particle.

\begin{tabular}{cccc}
\hline & Poisson's Ratio & Shear Modulus $\mathbf{( P a )}$ & Density $\mathbf{( k g / \mathbf { m } ^ { \mathbf { 3 } } )}$ \\
\hline Y-shaped elbow & 0.40 & $9.89 \times 10^{8}$ & 7800 \\
Particle & 0.22 & $2.13 \times 10^{7}$ & 2000 \\
\hline
\end{tabular}

The time step needs to be matched for the coupling calculation. Generally, the time step of Fluent was 10-100 times that of EDEM. Rayleigh time was used to evaluate the particles. When particles collide, $70 \%$ of the energy was consumed by the Rayleigh wave; therefore, the critical time step was determined by the velocity of the Rayleigh wave propagating through a solid particle. It is mainly affected by particle size, density and shear modulus of particles. The formula is:

$$
T_{R}=\frac{\pi R\left(\frac{\rho}{G}\right)}{(0.1631 \mu+0.8766)}
$$

where $R, \rho, G$ and $\mu$ represent the radius, density, shear modulus and Poisson's ratio of the particles, respectively. Of the $T_{R} 36 \%$ was set as the time step in EDEM and was $3 \times 10^{-5} \mathrm{~s}$ and fluent was $3 \times 10^{-4} \mathrm{~s}$.

\section{Results and Discussion}

The outlet flow rates of the simulation and experiment were compared to verify the validity of the model. From the Figure 7, the flow rates of the outlet obtained by the experiment was slightly less than the simulation, we believe the only reason is that the particle flow interferes with the measurement of the flow rate, thus resulting in an error. However, the difference can be negligible for the research in this paper.

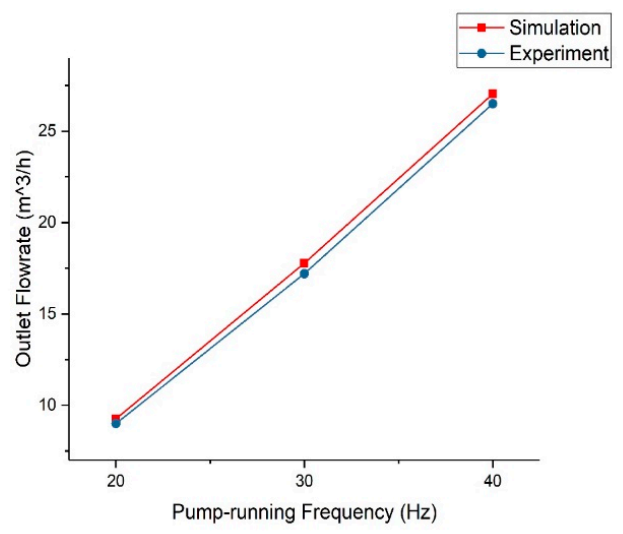

Figure 7. Comparison of the outlet flow rates obtained by the simulation and experiment. 


\subsection{Particle Analysis}

\subsubsection{Particle Distribution}

Particle distribution in the Y-shaped elbow obtained by simulation and experiment is shown in Table 6. Comparing the simulation and experimental results, it can be seen that the distribution and behavior of the particles in the mixing zone were basically the same. The simulation images show the results at $2.4 \mathrm{~s}$, because the distribution had reached a relatively stable state.

Table 6. Particle distribution in the Y-shaped elbow obtained by simulation and experiment.

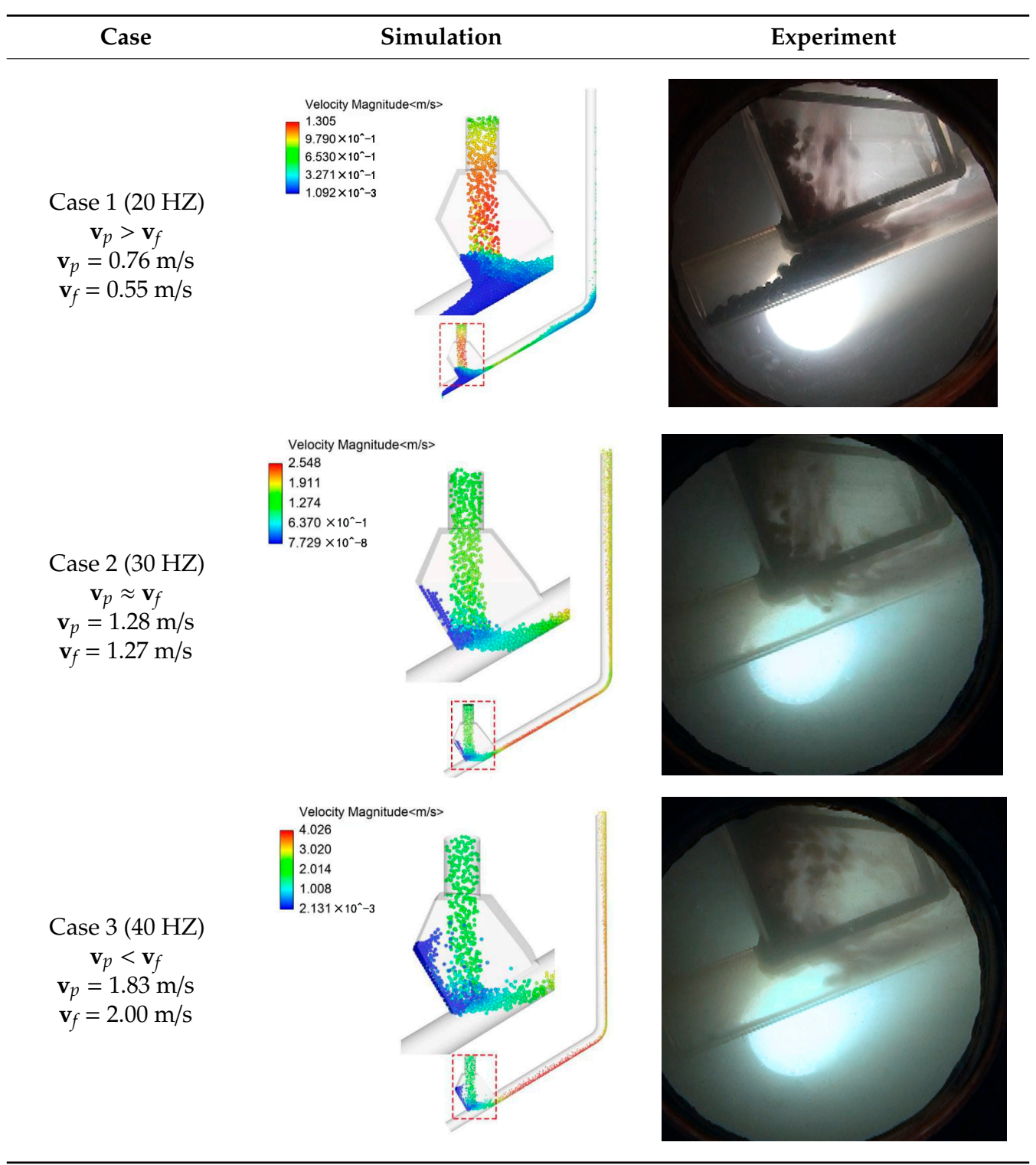

Although some of particles in the Y-shaped elbow were transported, stacking of particles or even leakage occurred at the same time in case 1 . In contrast, particles in case 2 and case 3 can be smoothly transported upwards. In our opinion, the reason for the stacking or leakage of particles in case 1 is that the fluid cannot dislodge the particles in time when the pump ran at a low frequency. From the perspective of forces of the particles, the thrust of the fluid flow on the particles was smaller than the component of the effective gravity of the particles parallel to the pipe wall, resulting in a downward trend of the particles. At this point, the particle velocity was greater than the fluid, and particles entering the pipe subsequently had a squeezing effect on the previous ones. It also exacerbated the 
tendency of particle stacking and leakage. As the pump running frequency increased, the increasing velocity in both inlets in case 2 and 3 eliminated the accumulation of particles. Meanwhile, the greater the fluid velocity at water inlet, the greater impact force on the particles; therefore, the location where particles collided with the pipe wall in case 3 was farther away from the lower end of the pipe. After particles entered the vertical lift pipe, they began to diffuse toward the pipe center.

Most of the particles in the above simulation conditions moved along the pipe wall basically. We believe that this phenomenon is caused by the gravity of large particles, the conveying velocity is not large to provide enough force to counteract the gravity component of the particles perpendicular to the inclined pipe. Additional simulations were implemented as follows in Figure 8 to make a comparison with case 1-3. The flow rate of particle inlet was doubled and the water inlet was 1,2 and 4 times that of case 3, respectively. With the increasing of conveying velocity, most of the particles no longer moved along the pipe wall. Meanwhile, particles entering the vertical pipe will diffuse to the pipe center more quickly. More particles were accumulated in the mixing chamber due to the impact of the high-speed water flow. Comparing the simulations under these three conditions, it can be found the particles in the inclined pipe become sparse as the flow rate of the water inlet increased. The main reason is that the impact of water on the particles increased as the water flow rate increased, more and more particles were pushed back into the mixing cavity; therefore, the flow rate of the water inlet cannot be too large, otherwise the efficiency of particle transportation will also be reduced. The particle motions will be discussed in detail later.

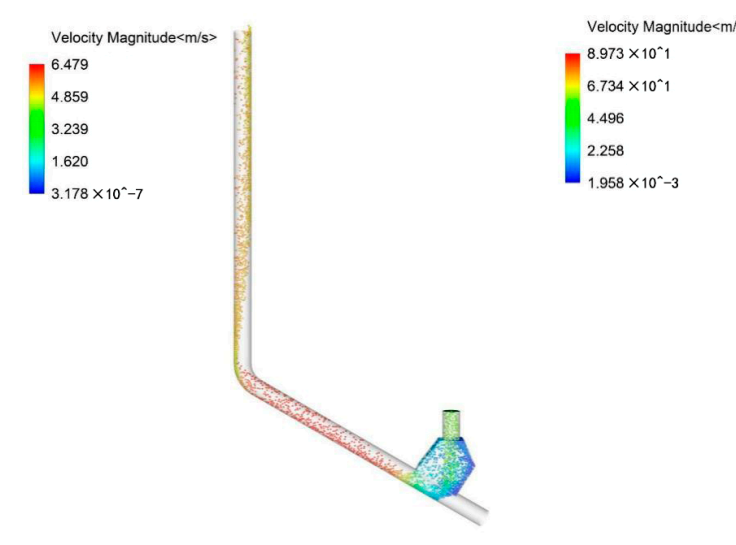

(a)

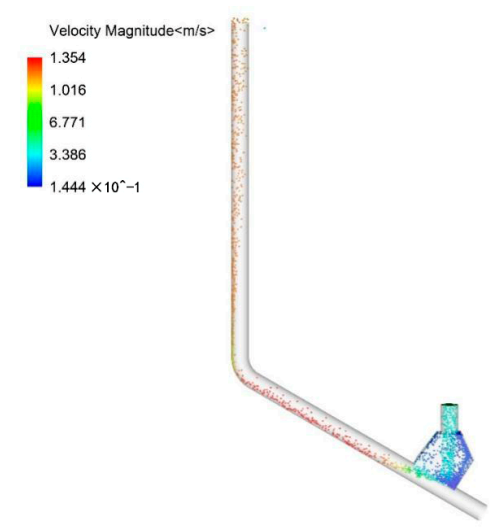

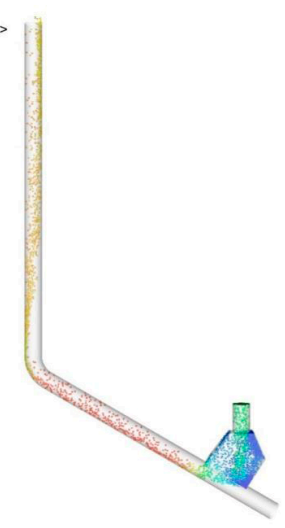

(b)

(c)

Figure 8. Additional simulations under higher conveying velocities: (a) $\mathrm{v}_{p}=4 \mathrm{~m} / \mathrm{s}, \mathrm{v}_{f}=2 \mathrm{~m} / \mathrm{s} ;(\mathbf{b}) \mathrm{v}_{p}=4$ $\mathrm{m} / \mathrm{s}, \mathrm{v}_{f}=4 \mathrm{~m} / \mathrm{s}$ and $(\mathrm{c}) \mathrm{v}_{p}=4 \mathrm{~m} / \mathrm{s}, \mathrm{v}_{f}=8 \mathrm{~m} / \mathrm{s}$. 


\subsubsection{Particle Motions}

Comparing the post-simulation animations and the experiments under three cases, we found that the movement state of the particles underwent a progressive change, shown in Figure 9. The particle motions became more complicated with the lifting speed increases. The sampling data analysis was performed on the particle groups. The particles with the same motion mode (50 particles) were selected. The following five main particle flow patterns were obtained: smooth delivery, postponed delivery, detention, stacked and leaked, which were defined as particle 1-5, respectively. The curves of particle velocity versus time in different patterns are shown in Figure 10 . Note that the velocity value " 0 " in the curve means that the particle is not in the calculation domain (not entering or having left). What is in common in Figure 10a is that particle 1 and 2 could be successfully transported. When particles were mixed with the fluid flow at the initial stage, the direction of particle flow changed. After a brief increase in velocity, it decayed due to collision with the inner wall of the pipe. It was found that the velocity of particle 1 underwent a secondary decay at the point where it entered the pipe elbow, after which it accelerated briefly and entered the vertical part of the Y-shaped elbow. As for particle 2, it took more time to entering the lifting pipe, because it was "trapped" in the mixing cavity due to the impact of fluid flow and other particles, but it finally entered the lifting pipe after an arc motion in the mixing cavity. The particle 1 and 2 mainly occurred in the cases of medium and high lifting velocities, while particle 3-5 mainly occurred in the cases of medium and low lifting velocities. As is shown in Figure 10b, the commonality among particle 3, 4 and 5 was that they could not make it to the outlet. Particle 3 was "trapped" in the mixing cavity and maintained a dynamic motion with a very small velocity, while particle 4 was stuck because of particle stacking in the pipe; therefore, the velocity of particle 4 was very close to zero. As for particle 5, it finally leaked out of the water inlet (the velocity peaked briefly at $1.8 \mathrm{~s}$ in the figure).

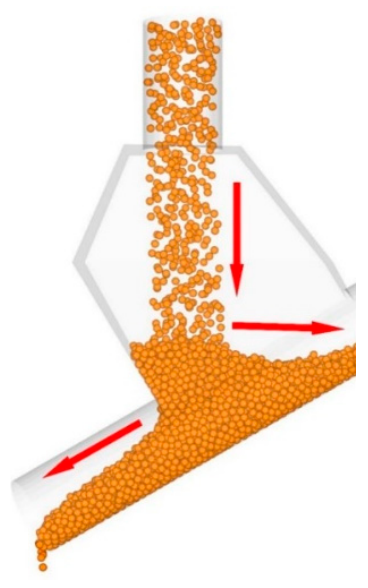

(a)

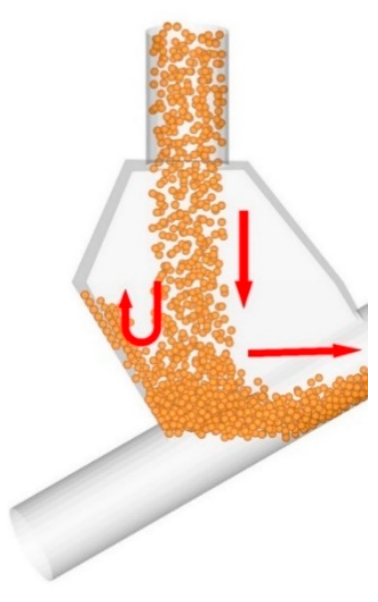

(b)

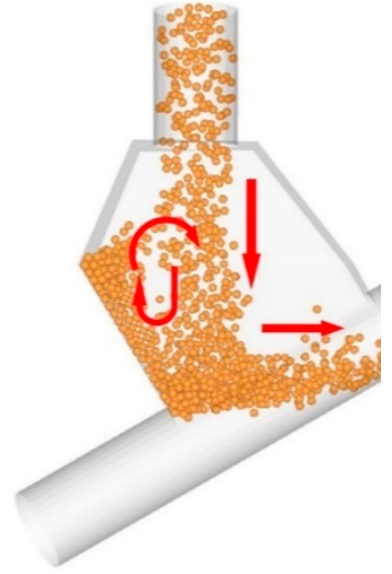

(c)

Figure 9. Progressive motions of particles in the mixing cavity under different cases. (a) Case 1; (b) Case 2 and (c) Case 3.

In general, the suggestions on the particle application based on the results above can be put forward. When the lift pump is soft-started (the conveying velocity is small), the particles will accumulate or leak, but the problems will be solved as soon as the pump speed increases. Adjusting the pump to the medium speed before start the feeding machine in the actual mining process will also help reduce the accumulation and leakage. At the same time, the relationship of the flow rate between particle inlet and water inlet should be balanced. For the simulation and experiment in this paper, it is proper that the flow rate of water inlet should be kept approximately 1-2 times that of the particle inlet. 


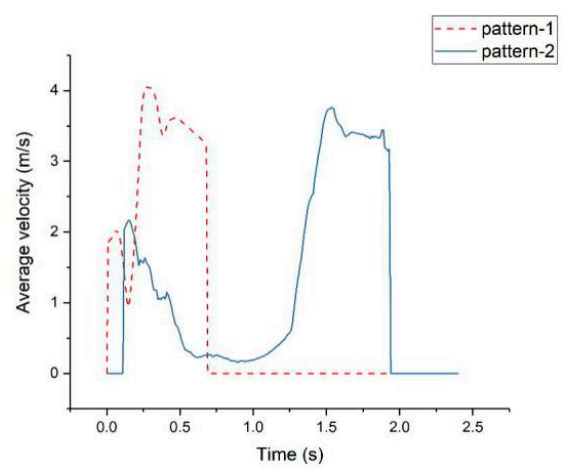

(a)

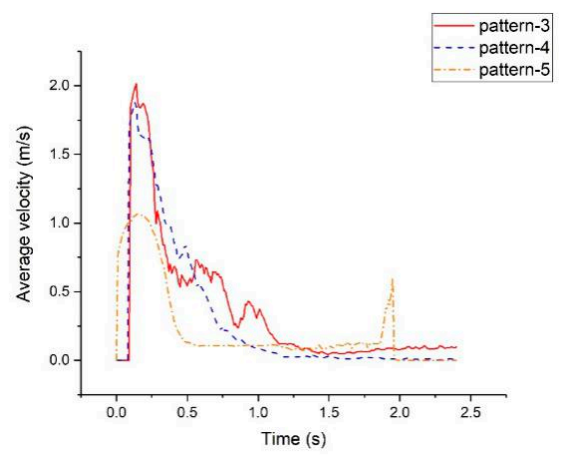

(b)

Figure 10. Different particle flow patterns under three cases. (a) Particles that can be transported and (b) particles that failed to transport.

\subsection{Flow Field}

Due to the small conveying velocity, the particles are prone to accumulation and leakage; therefore, it should be avoided in practical engineering applications. More attention should be paid on the working condition that particles can be smoothly transported. The detailed flow field information about case 3 are mainly shown and analyzed in this section.

\subsubsection{Contour of Velocity}

Figure 11 shows that the velocity distributions of fluid and particles in the Y-shaped elbows under case 3 were in the same state; that is, the distribution of the particles was substantially consistent with the high velocity region of the fluid in the pipe, except at the bend. The reason can be explained that the fluid near the intrados of the bend accelerated while near the extrados of the bend slowed down because of the effect of centrifugal force. As for particles, the gravity plays a major role in this situation. Most of particles move alone the pipe wall. When entering the bend, the particles continued to move along the pipe due to the centrifugal force. After the particles flowed into the vertical lifting pipe, they gradually diffused toward the pipe axis with the velocity increases. The fluid streamline in the mixing cavity was also made for a more detailed explanation in Figure 12. A vortex was formed due to the fluid interaction between particle and water inlets, which is why some of the particles will do an arc motion in the cavity.

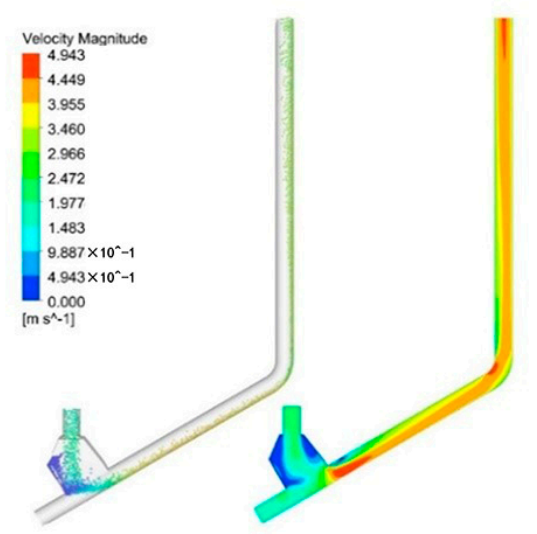

Figure 11. The velocity distributions of particles and fluid under Case 3. 


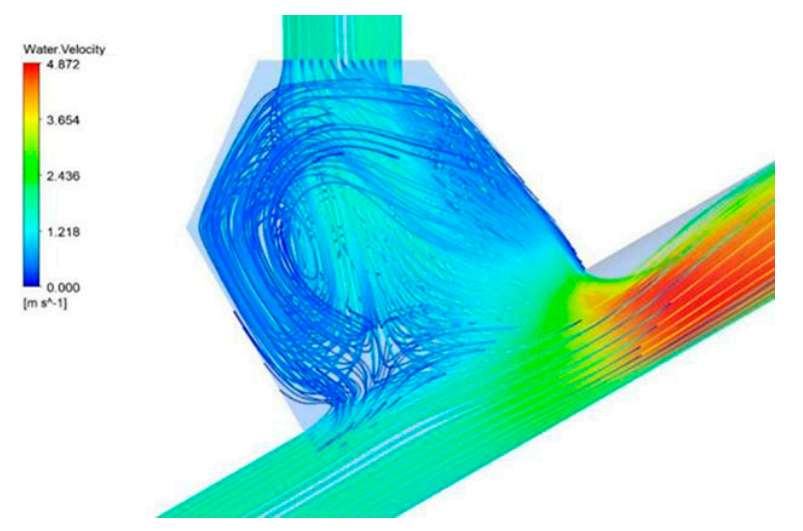

Figure 12. Velocity streamline in the mixing cavity under Case 3.

Additionally, average velocities of fluid and particles in the Y-shaped elbow were calculated, shown in Figure 13. The average velocities of fluid in case 2 and 3 were greater than that of particles. Analysis from the perspective of particle forces was performed. Large particles were mainly subjected to gravity, drag forces exerted by the fluid, and interaction forces with other particles in the fluid domain, particles keep sliding along the pipe for the fluid cannot provide enough lifting force to balance the gravity component of the particle parallel to the pipe wall. Consequently, the particle cannot fully accelerate; therefore, the fluid was faster than particles.

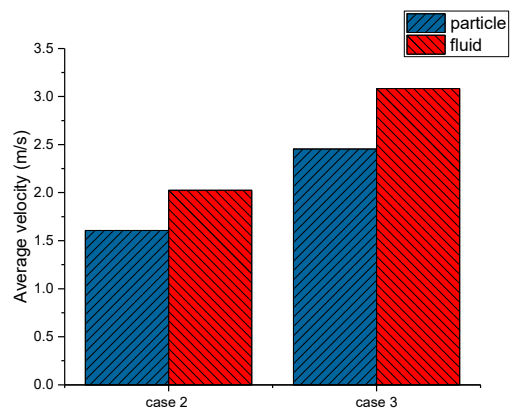

Figure 13. Average velocities of fluid and particles under Case 2 and 3.

\subsubsection{Secondary Flow}

The secondary flow of fluid plays an important role in particle motion and elbow erosion $[32,37,38]$. Here, the secondary flow paths and vectors of four red-cross sectional planes of the $120^{\circ}$ Y-shaped elbow in case 3 are observed in Figure 14. Due to the influence of fluid from the particle inlet, the flow direction of section a was towards the lower pipe wall, the fluid pushed the particles downward. Along the inclined pipe, the flow direction of section $b$ was the same as section a and the secondary flow also played a part in the movement of particles moving along the wall currently. The difference is that the bigger vortex moved to the center of the pipe. At section $c$, the vortex disappeared and the vectors flow from the extrados towards intrados of the elbow influenced by the centrifugal force. At the vertical part of the pipe, the flow direction of section $d$ changed back to the outside of the pipe wall. Still, particles that have just entered the vertical pipe were maintaining a state of moving along the wall while not diffusing instantly. On the one hand, they were mainly affected by the inertia, the influence by the secondary flow could not be ignored on the other hand. Based on the analysis and other papers, it could be inferred that large wear will occur on the extrados of the pipe, more attention will be paid on the effect of secondary flow and different working conditions on particle transport and pipeline wear in the future study. 


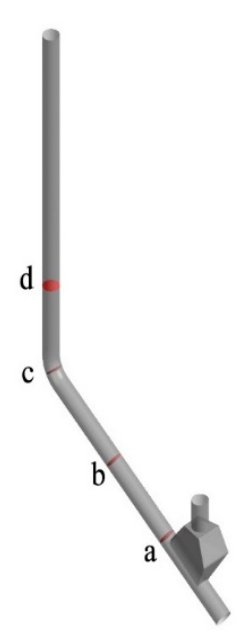

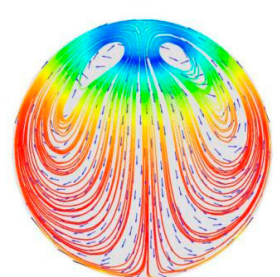

(a) velocity trace in cross-section a
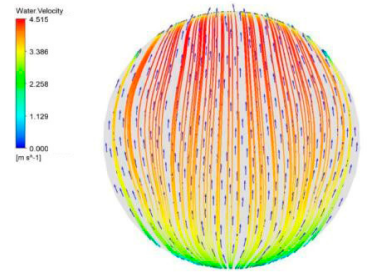

(c) velocity trace in cross-section c
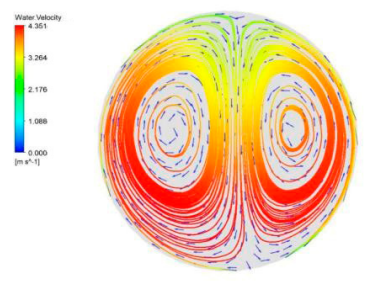

(b) velocity trace in cross-section b

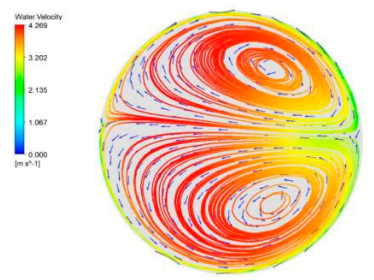

(d) velocity trace in cross-section d

Figure 14. Secondary flows of the Y-shaped elbow at different locations in Case 3.

\section{Conclusions}

Simulation analysis based on CFD-DEM coupled calculations of the particle-liquid flow in the Y-shaped elbow was performed. Various results under different working conditions were compared with the experimental results. The main conclusions can be drawn as follows:

The conveying velocity played a leading role in transporting particles. When the pump ran at low frequencies, the flow rates of both inlets was small, most of the particles would stack and leak out of the water inlet. However, increasing the velocity applied by the lift pump would improve the transmission efficiency. However, the extra simulations at a larger flow rate also demonstrated that the flow rate of water inlet could not be too large, because more particles would be "trapped" in the mixing cavity for the impact of water. Therefore, it is recommended that water velocity applied by the lifting pump be 1-2 times that of particles applied by the feeding machine in engineering applications. The velocity of particles in the flow field was generally less than the fluid velocity. Large particles kept moving along the pipe wall due to gravity and the secondary flows of the fluid. With the increasing of conveying velocities, the particles in the pipe will spread more thoroughly and the movement will be more complicated.

Author Contributions: The manuscript was written by Q.H. and L.Z.; all authors discussed the original idea; conceptualization, Q.H. and T.L.; methodology, L.Z.; software, Q.H.; validation, Q.H., L.Z. and T.S.; formal analysis, Y.G.; investigation, T.L.; resources, T.S.; data curation, Q.H.; writing一original draft preparation, L.Z.; writing-review and editing, Q.H.; visualization, T.L.; supervision, T.S.; project administration, L.Z.; funding acquisition, L.Z. All authors have read and agreed to the published version of the manuscript.

Funding: This research was funded by National Key Research and Development Program of China (2019YFC0312400).

Acknowledgments: Deep-sea Polymetallic Nodule Mining Test Project (2016YFC0304101-8).

Conflicts of Interest: The authors declare no conflict of interest.

\section{References}

1. Liu, Y.B.; Chen, J.Z.; Yang, Y.R. Numerical simulation of liquid-solid two-phase flow in slurry pipeline transportation. J. Zhejiang Univ. Eng. Sci. 2006, 40, 858-863.

2. Kaushal, D.R.; Thinglas, T.; Tomita, Y.; Kuchii, S.; Tsukamoto, H. CFD modeling for pipeline flow of fine particles at high concentration. Int. J. Multiph. Flow. 2012, 43, 85-100. [CrossRef] 
3. Ravikumar, S.K.; Ziazi, R.M.; Chambers, F.W.; McNally, M.E.; Hoffman, R.M. Computational prediction of particle-laden slurry flow in a vertical pipe using Reynolds Stress Model. In Fluids Engineering Division Summer Meeting; American Society of Mechanical Engineers: New York, NY, USA, 2013; Volume 55560, p. V01CT20A008.

4. Li, M.; He, Y.; Liu, Y.; Huang, C. Hydrodynamic simulation of multi-sized high concentration slurry transport in pipelines. Ocean Eng. 2018, 163, 691-705. [CrossRef]

5. Messa, G.V.; Malavasi, S. A CFD-based method for slurry erosion prediction. Wear 2018, 398, 127-145. [CrossRef]

6. Qiang, Z. Simulation Analysis of Sinking Process of Particles Falling into Water Based on Coupled CFD-DEM. Master's Thesis, Jilin University, Jilin, China, 2014.

7. Parsi, M.; Kara, M.; Agrawal, M.; Kesana, N.; Jatale, A.; Sharma, P.; Shirazi, S. CFD simulation of sand particle erosion under multiphase flow conditions. Wear 2017, 376, 1176-1184. [CrossRef]

8. Huang, S.; Shu, Y.L.; Zhou, J.J.; He, D.P.; Peng, T.Y. Numerical simulation for solid-liquid two-phase flow in discharge pipeline and flange connection by CFD-DEM coupling. Hydromechatronics Eng. 2017, 12, 1-6.

9. Kannojiya, V.; Deshwal, M.; Deshwal, D. Numerical Analysis of Solid Particle Erosion in Pipe Elbow. Mater. Today Proc. 2018, 5, 5021-5030.

10. Cundall, P.A.; Strack, O.D.L. A discrete numerical model for granular assemblies. Géotechnique 1979, 29, 47-65. [CrossRef]

11. Chen, X.; Zhong, W.; Zhou, X.; Jin, B.; Sun, B. CFD-DEM simulation of particle transport and deposition in pulmonary airway. Powder Technol. 2012, 228, 309-318. [CrossRef]

12. Capecelatro, J.; Desjardins, O. An Euler-Lagrange strategy for simulating particle-laden flows. J. Comput. Phys. 2013, 238, 1-31. [CrossRef]

13. Park, K.M.; Yoon, H.S.; Kim, M.I. CFD-DEM based numerical simulation of liquid-gas-particle mixture flow in dam break. Commun. Nonliner Sci. 2018, 59, 105-121. [CrossRef]

14. Huang, S.; Su, X.; Qiu, G. Transient numerical simulation for solid-liquid flow in a centrifugal pump by DEM-CFD coupling. Eng. Appl. Comp. Fluid. 2015, 9, 411-418. [CrossRef]

15. Kloss, C.; Goniva, C.; Hager, A.; Amberger, S.; Pirker, S. Models, algorithms and validation for opensource DEM and CFD-DEM. Prog. Comput. Fluid Dyn. 2012, 12, 140-152. [CrossRef]

16. Blais, B.; Bertrand, O.; Fradette, L.; Bertrand, F. CFD-DEM simulations of early turbulent solid-liquid mixing: Prediction of suspension curve and just-suspended speed. Chem. Eng. Res. Des. 2017, 123, 388-406. [CrossRef]

17. Uzi, A.; Levy, A. Flow characteristics of coarse particles in horizontal hydraulic conveying. Powder Technol. 2018, 326, 302-321. [CrossRef]

18. Park, Y.C.; Yoon, C.H.; Lee, D.K.; Kwon, S.K. Experimental Studies on Hydraulic Lifting of Solid-liquid Two-phase Flow. Ocean Polar Res. 2004, 26, 647-653. [CrossRef]

19. Chen, C.G.; Yang, Y.; Tang, D.S.; Jin, X.; Xiao, H. Study on the settling regularity of solid particles in vertical pipelines. Int. J. Sediment Res. 2010, 4, 16-21.

20. Wang, Y.; Yang, N.; Jin, X. Study on the Motion Path of Solid Particle in Vertical Pipeline. Jinshu Kuangshan/Met. Mine 2011, 40, 36-39.

21. Vittorio Messa, G.; Malavasi, S. Numerical prediction of particle distribution of solid-liquid slurries in straight pipes and bends. Eng. Appl. Comput. Fluid Mech. 2014, 8, 356-372. [CrossRef]

22. Wijk, J.M.V.; Talmon, A.M.; Rhee, C.V. Stability of vertical hydraulic transport processes for deep ocean mining: An experimental study. Ocean Eng. 2016, 125, 203-213. [CrossRef]

23. Ekambara, K.; Sanders, R.S.; Nandakumar, K.; Masliyah, J.H. Hydrodynamic Simulation of Horizontal Slurry Pipeline Flow Using ANSYS-CFX. Ind. Eng. Chem. Res. 2009, 48, 8159-8171. [CrossRef]

24. Zhang, H.; Chen, L.; Xie, R.; Liu, X.; Zheng, X.; Shang, Z. Numerical simulation of solid-liquid two-phase steady flow in horizontal pipe. J. Chem. Ind. Eng. Soc. China 2009, 5, 1162-1168.

25. Capecelatro, J.; Desjardins, O. Eulerian-Lagrangian modeling of turbulent liquid-solid slurries in horizontal pipes. Int. J. Multiph. Flow 2013, 55, 64-79. [CrossRef]

26. Messa, G.V.; Malin, M.; Malavasi, S. Numerical prediction of fully-suspended slurry flow in horizontal pipes. Powder Technol. 2014, 256, 61-70. [CrossRef]

27. Singh, J.P.; Kumar, S.; Mohapatra, S.K. Modelling of two phase solid-liquid flow in horizontal pipe using computational fluid dynamics technique. Int. J. Hydrogen Energy 2013, 42, 20133-20137. [CrossRef] 
28. Messa, G.V.; Malavasi, S. Numerical prediction of dispersed turbulent liquid-solid flows in vertical pipes. J. Hydraul. Res. 2014, 52, 684-692. [CrossRef]

29. Kaushal, D.R.; Kumar, A.; Tomita, Y.; Kuchii, S.; Tsukamoto, H. Flow of Bi-modal Slurry through Horizontal Bend. Kona Powder Part. J. 2017, 34, 258-274. [CrossRef]

30. Oka, Y.I.; Okamura, K.; Yoshida, T. Practical estimation of erosion damage caused by solid particle impact. Wear 2005, 259, 95-101. [CrossRef]

31. Cao, B.; Xia, J.X.; Hei, P.F.; Liu, X. Study on the Movement State of Coarse-grain during Hydraulic Transportation in Pipeline of Complex Spatial Morphology. Min. Met. Eng. 2012, 2, 5-10.

32. Chen, J.K.; Wang, Y.S.; Li, X.F.; He, R.Y.; Han, S.; Chen, Y.L. Erosion prediction of liquid-particle two-phase flow in pipeline elbows via CFD-DEM coupling method. Powder Technol. 2015, 282, 25-31. [CrossRef]

33. Peng, W.; Cao, X. Numerical simulation of solid particle erosion in pipe bends for liquid-solid flow. Powder Technol. 2016, 294, 266-279. [CrossRef]

34. Masanobu, S.; Takano, S.; Fujiwara, T.; Kanada, S.; Ono, M.; Sasagawa, H. Study on Hydraulic Transport of Large Solid Particles in Inclined Pipes for Subsea Mining. J. Energy Resour. Technol. 2017, 139. [CrossRef]

35. Zhu, H.P.; Zhou, Z.Y.; Yang, R.Y.; Yu, A.B. Discrete particle simulation of particulate systems: Theoretical developments. Chem. Eng. Sci. 2007, 62, 3378-3396. [CrossRef]

36. Chu, K.W.; Chen, J.; Yu, A.B. Applicability of a coarse-grained CFD-DEM model on dense medium cyclone. Min. Eng. 2016, 90, 43-54. [CrossRef]

37. Alletto, M.; Breuer, M. Prediction of turbulent particle-laden flow in horizontal smooth and rough pipes inducing secondary flow. Int. J. Multiph. Flow. 2013, 55, 80-98. [CrossRef]

38. Jia, M.; Yan, C.; Song, J.; Wei, Y.; Zhou, F.; Sun, L.; Wang, D. Experimental and numerical study of secondary flow in a T-type bend of a CFB riser. Chem. Eng. J. 2018, 334, 1222-1232. [CrossRef]

(C) 2020 by the authors. Licensee MDPI, Basel, Switzerland. This article is an open access article distributed under the terms and conditions of the Creative Commons Attribution (CC BY) license (http://creativecommons.org/licenses/by/4.0/). 